\title{
L'enrichissement du modèle français au contact d'autres modèles : cas de l'Angleterre
}

\author{
Organisation of water services in France and Great Britain - a comparison
}

par H. Speed

Chairman, Northumbrian Water Limited

The paper describes the organisation of water services in France, where arrangements differ from these of England and Wales, Scotland and Northern Ireland. The regulation of these services is described and thereafter the problems of the present and future are discussed.

\section{I INTRODUCTION}

Même si l'on parle de "Royaume Uni", l'organisation des services des eaux dans les trois pays est complètement différente.

- En Angleterre et au Pays de Galles, les Compagnies Eaux et Assainissement (Water Companies) sont privatisées à $100 \%$. Cette privatisation date de 1989 , quand les dix anciens Services des Eaux étaient mis en vente par le gouvernement à la Bourse londonienne. A cette époque, $25 \%$ de l'eau potable était fournie par quelque 28 compagnies des eaux privées, dont la plupart existaient depuis le début du $\mathrm{XIX}^{\mathrm{e}}$ siècle. La loi sur l'eau votée en 1989 qui a autorisé le gouvernement à vendre les Services des Eaux, a permis à ces compagnies d'échanger les lois restrictives qui les avaient gouvernées contre celles d'une SARL normale. La plupart d'entre elles ont adopté ces lois.

- En Écosse, la distribution d'eau, qui, jusque là, était assurée par l'administration locale, a été transférée le premier avril 1996, à trois nouveaux Services des Eaux et Assainissement.

- En Irlande du Nord, le Département de l'Environnement se charge des services eaux et assainissement.

Dans ce papier ne seront traités que les cas de l'Angleterre et du Pays de Galles.

\section{II — L'ORGANISATION EN ANGLETERRE ET AU PAYS DE GALLES}

(population 50 millions)

\subsection{Vue d'ensemble/préambule}

Parmi les dix SARL eau et assainissement (Water plc's) créées en 1989, deux d'entre elles, la Welsh et la North-West ont acquis des compagnies de distribution régionale d'électricité et sont désormais appelées des "multi-utilities". Une troisième société, la Southern, a été rachetée par une compagnie d'électricité, le Scottish Power, qui en sus de ses activités de génération et de distribution d'électricité, fournit du gaz et est active dans les télécommunications. Une quatrième société, la Northumbrian, appartient à part entière à la Suez Lyonnaise des Eaux.

Les 28 SARL qui se spécialisaient dans la distribution d'eau potable ont subi des changements elles aussi. Aujourd'hui elles sont 19, suite à des O.P.A. et des fusions. Peu d'entre elles sont restées indépendantes, la plupart sont détenues en partie ou en entier par des groupes plus importants (y compris les trois groupes français, la Générale des Eaux, Suez Lyonnaise des Eaux et la Saur) ou par des investisseurs.

Le gouvernement britannique a gagné 5 milliards de livres par la vente des Services Eau et Assainissement, qui ont été utilisés pour l'amortissement de leur créances qui totalisaient quelque 5 milliards de livres. En plus il les a dotés de 7,7 milliards de crédit d'impôts et d'une subvention d'1,5 milliard de livres (la "dot verte") censée financer des projets de protection de l'environnement.

Tous les Water plcs et plusieurs des SARL qui se spécialisent dans la distribution d'eau, font sous-traiter la distribution d'eau et/ou l'assainissement par des filiales qui sont soumises à des règlements et connues sous le nom de "core businesses". Ces entreprises réglées sont donc les filiales d'une compagnie mère, qui, avec d'autres filiales commerciales ne sont pas soumises à la réglementation.

Toutes les "core businesses" opèrent sous le régime d'une licence à durée de 25 ans limitée à une région géographique 
déterminée. Cette licence peut se renouveler, mais peut également être annulée par le Secrétaire d'état pour l'Environnement si la compagnie manque dans ses obligations de fournisseur. La licence peut également être soumise à des changements à la demande du régulateur, OFWAT, (voir plus loin) afin de permettre à d'autres fournisseurs de distribuer de l'eau dans quelques secteurs géographiques particuliers, appelés "insets".

Cette possibilité d'insérer/introduire d'autres fournisseurs dans cette structure, dont la caractéristique la plus frappante est la monopolisation, permet de faire jouer un peu la concurrence.

La législation prévoit également aux "core businesses" de fournir des clients avoisinants quand une compagnie peut se montrer plus compétitive que sa voisine.

\subsection{La réglementation}

Tout système qui fournit des services au public doit être appuyé par un système de régulation. Dans la plupart des cas, plus les propriétaires sont éloignés de leurs clients, plus la réglementation est sévère.

Ainsi, ce n'est pas étonnant que le système de réglementation le plus complet et le plus complexe soit opérationnel en Angleterre et dans le pays de Galles - puisque ces derniers sont les seuls pays du monde dont les compagnies d'eau sont détenues à $100 \%$ par des investisseurs privés.

Trois régulateurs indépendants ont été créés au moment de la privatisation en 1989, à savoir :

- I'OFWAT: Office of Water Services - Le Bureau des Services d'Eau

- Le DWI : Drinking Water Inspectorate - L'Inspectorat de l'Eau Potable

- La NRA : National Rivers Authority - L'Autorité Nationale des Rivières qui fait partie de l'Agence de l'Environnement (EA) depuis le premier avril 1996.

\subsection{L'OFWAT}

Le Bureau des Services d'Eau est souvent appelé le "régulateur économique". Ses fonctions concernent :

- l'établissement des tarifs,

- le suivi des performances,

- les relations clientèle, en partie à travers des Comités de Relations Clientèles.

\subsubsection{Etablissement des tarifs}

Le prix de l'eau et de l'assainissement est calculé tous les ans selon la formule rpi $\pm x$ où rpi est le taux annuel d'inflation pour le mois de novembre, publié au mois de décembre. $x$ est un rajustement positif ou négatif basé sur les besoins d'investissement et les buts d'efficacité de chaque compagnie.

L'OFWAT donne le valeur d' ' $x$ ', valable normalement pendant dix ans, mais jusqu'ici il y a une révision tous les 5 ans, pour chaque compagnie.

Ces valeurs sont basées à la fois sur les résultats dans le passé et les projections dans l'avenir des investissements et les dépenses (de revenu) avec des hypothèses quant aux améliorations d'efficacité.

Cette forme de contrôle des tarifs est souvent appelée réglementation "price cap". L'idée est d'encourager des entreprises à améliorer leur efficacité de façon à garder autant que possible les revenus autorisés par l'OFWAT. Cela s'oppose à la réglementation "rate of return" ou "taux de retour" d'utilisation très répandue aux États-Unis. Ce système applique un taux de retour (autour des 10\%) aux investissements et amène souvent au surinvestissement et donc à l'inefficacité.

\subsubsection{Le suivi des résultats}

Au mois de juillet, chaque compagnie soumet à I'OFWAT des données détaillées sur ses résultats. Ces données sont analysées afin d'assurer que :

- les programmes d'investissement sont en route,

- les investissements produisent les résultats escomptés,

- le niveau du service clientèle est maintenu ou amélioré.

\subsubsection{Service clientèle}

L'OFWAT est chargé d'établir le niveau du service clientèle, non seulement pour les niveaux de pression et de flux chez le client, mais aussi pour d'autres mesures de service tels :

- la quantité de plaintes pour eau de couleur anormale,

- la quantité de coupures de distribution,

- la vitesse de réponse aux appels téléphoniques et au courrier,

- la fiabilité des rendez-vous donnés,

- les problèmes de débordement des eaux usées, et ainsi de suite...

Des tableaux comparatifs de ces données sont publiés tous les ans et les clients qui n'ont pas reçu la qualité de service promise sont indemnisés automatiquement.

A l'intérieur de chacun des dix domaines des services d'eau et d'assainissement, il existe une Comité de Service Clientèle. Ces comités s'occupent des plaintes, font des observations sur les problèmes des consommateurs au niveau local et rendent compte à l'OFWAT.

\subsubsection{Le D.W.I}

L'Inspectorat de l'Eau Potable est une division du Département de l'Environnement. Son rôle consiste à suivre la qualité physique et bactériologique de l'eau potable.

Pour ce faire, une fois par an il vérifie sur site les données analytiques des compagnies et leurs laboratoires. Plusieurs aspects sont mis à l'examen, y compris le niveau de qualification, de compétence et de formation du personnel des laboratoires et des stations d'épuration. Les stations et les réservoirs sont inspectés à leur tour.

Des rapports annuels sont édités. Ils sont dans le domaine public, comme le sont toutes les données traitant de la qualité d'une entreprise.

Les compagnies doivent rendre compte des événements tels les pannes de chloration, ou la présence de coliformes fécaux dans des échantillons. Le DWI a le pouvoir de poursuivre en justice les compagnies et il l'exerce (librement).

\subsubsection{L'Agence de l'Environnement}

La privatisation des dix services d'eau et d'assainissement a nécessité la création d'une nouvelle organisation chargée de contrôler la gestion des bassins versants, comprenant l'allocation des ressources et la maîtrise de la pollution. Ces fonctions qui étaient acquittées par les dix services d'eau avant leur privatisation étaient désormais assumées par la 
NRA - I'Autorité Nationale des Rivières. La NRA à son tour fait partie de la nouvelle Agence de l'Environnement depuis sa création le premier avril 1996.

\section{III $\square$ DISCUSSION}

De ce qui précède, il est évident que l'organisation des "water services" en Grande-Bretagne est complexe et diffère considérablement de celle de la France. Ce qui ressort moins des portraits précédants, est l'envergure/importance de l'attention que l'industrie a attiré aux niveaux politique, public et médiatique dans les deux pays ces dernières années.

Alors que les entreprises fournisseurs d'eau en France ont reçu beaucoup d'attention de la part des médias depuis deux/trois ans, ceci paraît presque insignifiant comparé à la surveillance rapprochée, incessante, infligée sur les "water services" anglais et gallois. II y a plusieurs raisons à cela. D'abord, les "water services" ont été victimes de circonstances hors leur contrôle (telles de longues périodes de sécheresse conduisant à des restrictions/ruptures d'approvisionnement). Ensuite, la mauvaise presse dont ils ont souffert a été auto-infligée (des augmentations faramineuses de salaire, des "share options" très généreux ou des diversifications désastreuses.).

C"était la vente ("sell off") des biens publics en Angleterre et au Pays de Galles qui a provoqué beaucoup de ressentiment. Cette privatisation qui a creusé une différence fondamentale entre l'organisation en Angleterre et au Pays de Galles et celle en France. Malgré sept ans de possession privée (ou plus de 150 ans, dans le cas de bon nombre de "water only companies") en ce qui concerne les Britanniques, l'eau est un bien public dont la possession ne devrait pas relever du secteur privé. D’après ses expériences dans d'autres pays. l'auteur est convaincu que la gestion déléguée", telle qu'elle est pratiquée en France, sous ses formes diverses, est "vendable" et acceptable, mais que la vente de l'infrastructure provoque des émotions beaucoup plus négatives.

Le gouvernement était soucieux que le lancement de la privatisation réussisse face à une opposition considérable, à la fois de nature politique et environnementale et pour cette raison les termes de vente étaient peut-être trop généreux. Les facteurs d'ajustements de prix (facteur K) pendant les cinq premières années étaient établis par le gouvernement et ils ont assuré une forte rentabilité à une époque où la conjoncture économique du pays était relativement mauvaise.

Les actions des compagnies des eaux ont surpassé les "stock market indexes" de la Bourse, les dividendes étaient généreux et souvent les conseils d’administration se sont récompensés avec des augmentations de salaire considérables. Au début, au moins, ceci était un reflet des différentes façons d'opérer entre le secteur public et le secteur privé, mais les médias ont appelé les directeurs des compagnies des eaux des "fat cats" - de gros richards - une étiquette qui colle toujours.

Depuis la révision des prix à la fin des cinq premières années, les résultats financiers ont continué à être très bons. Le régime de régulation rémunère des entreprises pour leurs programmes d'investissement. L'intervalle de cinq ans entre révisions sert à éviter des opérations à court terme - c'est-àdire, des interventions fréquentes du régulateur. Néanmoins, plusieurs entreprises semblent être en retard dans leur programme d'investissement, mais continuent d'augmenter leurs prix tous les ans. Cela a conduit à une recrudescence de critiques du public. Tout comme dans la période qui précédait les élections présidentielles en France en 1995, quand les contrats publics étaient mis en vedette, ainsi en Angleterre, les partis de l'opposition ont attaqué avec force l'industrie de l'eau juste avant les élections législatives de 1997, la traitant d'un "gâchis de la part du parti conservateur". La sécheresse et des conditions météorologiques difficiles ont renforcé cet avis en soulignant les niveaux élevés de fuites, le manque de prévisions et un sous-investissement par quelques-unes des compagnies eau et assainissement.

\section{IV — L'AVENIR}

Pendant ce temps, le prix de l'eau a continué d'augmenter de façon brusque - avec des augmentations de plus de $40 \%$ de moyenne pendant les cinq premières années après la privatisation en Angleterre et au Pays de Galles. En partie ces augmentations devaient satisfaire au besoin réel d'investir. Après des années sans fonds en tant que service public, les nouvelles compagnies devaient désormais se conformer aux règlements européens stricts sur la qualité de l'eau potable et les eaux usées ("sewage discharge"). Ces règlements ont augmenté les prix en France aussi. Pour la plupart des clients, l'investissement accru n'a rien produit de concret et les changements sont invisibles. Pour les groupes de pression tels les "surfeurs contre les eaux usées", il existe toujours des plages polluées, bonnes à critiquer, même si la situation s'améliore rapidement.

Les compagnies dans les deux pays, ont essayé d'améliorer leur image avec des campagnes de relations publiques et des projets revalorisants dans les régions avoisinantes. Cependant la réaction du public vis-à-vis de ces campagnes de publicité est négative : les clients n'aiment pas qu'on dépense de l'argent sur la publicité. L'industrie de l'eau a donc une tâche énorme devant elle, si elle veut améliorer son acceptabilité et sa crédibilité - et ceci s'applique aux Français comme aux Anglais.

Les compagnies ont évolué lentement d'une attitude peu consciente des besoins du client, vers des entreprises dotées de service "relations clientèle", mais la plupart ont maintenant adopté le dicton que "le client est roi". Aujourd'hui dans la majorité d'entre elles, la réponse aux besoins du client et les niveaux de service et de qualité sont très bons. Cependant, il est difficile d'en convaincre le client, qui reste méfiant et lit constamment du mal concernant ces entreprises dans les journaux.

II est clair que, dans l'avenir, la réglementation dans le Royaume Uni qui gouverne le niveau de service requis et le revenu disponible, va se resserrer indépendemment du parti au pouvoir. Tout indique que la réglementation en vigueur en France va se resserrer aussi, à cause du besoin d'observer le règlement européen, de l'avis des hommes politiques que être dur avec les monopoles est populaire, et parce que les clients sont mieux informés et plus exigeants. Les compagnies des eaux en France devraient porter attention à ces développements et se préparer dès maintenant !

Dans les années à venir, gagner la confiance et l'estimation des clientes va constituer une tâche énorme. Ce dont les compagnies des eaux ont le plus besoin est une période de 
stabilité, loin des attaques médiatiques, pour que les souvenirs aient le temps de s'effacer quelque peu. Pour nombre de compagnies des eaux britanniques, quelques années de pluie abondante les aideraient pendant qu'elles règlent les problèmes de ressources. Cela préoccupe moins les compagnies des eaux en France, et les clients se montrent moins mécontents qu'en Grande-Bretagne. Les compagnies des eaux et leurs clients n'ont pas besoin d'une législation européenne encore plus lourde, qui nécessiterait des investissements plus grands et donc des augmentations pénibles de prix pour un gain de qualité imperceptible. Les utilities devraient se réunir afin de résister à des propositions législatives dans ce genre émanant de Bruxelles.

\section{LE MODÈLE FRANÇAIS : QUELQUES LEÇONS À TIRER DE L'EXPÉRIENCE BRITANNIQUE}

Sans aucune doute, le programme de privatisation initié par Margaret Thatcher a suscité l'intérêt du monde entier et a encouragé le secteur privé à participer à l'investissement dans les services d'utilité publique de façon très répandue. En ce qui concerne l'eau, l'idée thatcherienne de vendre les infrastructures n'a été ni très populaire ni beaucoup imitée. En revanche, les concepts britanniques de réglementation se sont avérés très populaires. Plusieurs gouvernements et états se sont rendus compte que pour réussir la délégation de la gestion des services il faut contrôler de près les tarifs, la qualité de l'eau et le niveau de service. Partout dans le monde dans les contrats de gestion déléguée on trouve des éléments de contrats basés sur le système britannique. Ces contrats ont souvent été rédigés selon les conseils de conseillers juridiques et financiers et des consultants britanniques.

Les sociétés françaises qui ont investi dans les water companies britanniques depuis 1988 ont certainement acquis une expérience de valeur de l'environnement réglementaire rigoureuse en vigueur en Angleterre et au Pays de Galles. A noter que la Générale des Eaux et la Saur se sont limitées au rachat de "water only companies" et ont finalisé leurs O.P.A. avant la fin 1989, alors que la Suez Lyonnaise des Eaux est allée plus loin par son acquisition d'une des dix compagnies eau et assainissement, la Northumbrian.

Leur expérience avec le système de réglementation "anglais" a certes aidé les compagnies françaises qui ont cherché des contrats au niveau mondial. Leurs filiales britanniques constituent un fonds de savoir-faire dont elles peuvent profiter quand elles travaillent dans des pays qui ont adopté des modèles anglo-saxons.
Les réglementations ont entraîné la nécessité qu'une attention particulière soit portée au client en Angleterre et au Pays de Galles. Ce souci et son système de soutien sont sans doute les plus avancés où que ce soit dans le monde pour les services des eaux. Encore une fois, cette expérience s'est avérée très utile en France où les demandes d'information et les exigences pour un niveau de service amélioré de la part du client averti ont augmenté énormément ces dernières années. Il y a quelques années, on affirmait en France que le client était le Maire, et en termes absolus, ceci est vrai. Cependant, l'intérêt et la prise de conscience des clients ont tellement augmenté que les individus qui utilisent les services des eaux s'attendent à parler directement avec leur service public. Le modèle français ne perdrait pas de sa souplesse si le client devait prendre plus d'importance de façon à mieux répondre à la demande interne et celle des marchés au niveau mondial.

Jusqu'à la privatisation en 1989 , tous les groupes de distribution d'eau avaient contribué à un programme national de recherche concerté. A partir de 1980, plus de la moitié de la recherche s'est concentrée sur les problèmes de corrosion des tuyaux en fer de fonte et les égouts en cours de détérioration. La recherche nationale concertée a été largement réduite après 1989 et s'est concentrée sur des sujets tels la réglementation et des problèmes de santé - qui n'entraînaient pas des questions de compétitivité parmi les sociétés.

Alors que les grands groupes français sont, selon toutes probabilités, plus avancés en ce qui concerne leurs recherches dans la technologie de traitement de l'eau et de l'eau usée, dans le Royaume-Uni les recherches dans les technologies de réhabilitation et remplacement des conduites et égouts sont plus avancées. Ces recherches comprennent le domaine des méthodes de pose de conduites sans terrassement "no dig" et "trenchless".

En France, les communes sont dans la grande majorité des cas, elles-mêmes les propriétaires des infrastructures et peu d'argent a été dépensé dans ces biens invisibles. Avec le temps, les systèmes ont vieilli et la qualité de l'eau dans les conduites a donc été détériorée ; il va falloir investir de plus en plus dans les infrastructures souterraines. Les groupes français pourront profiter du cumul de connaissances et d'expérience acquises dans le Royaume-Uni. De plus, des contrats internationaux majeurs tels ceux de Buenos Aires et Manille, nécessitent un travail d'envergure sur les conduites d'eau et les égouts - l'expérience des Britanniques pourrait être d'une grande valeur pour les services publics français, qu'ils travaillent uniquement en France ou à l'étranger. 